\title{
Hidratación inicial del cemento. Efecto de aditivos superplastificantes
}

\author{
Early hydration cement. Effect of \\ admixtures superplasticizers
}

F. PUERTAS Y T.VÁZQUEZ

Fecha de recepción: 27-XI-00

Instituto de Ciencias de la Construcción Eduardo Torroja(CSIC)

Fecha de aceptación: 20-II-01

ESPAÑA

\section{RESUMEN}

En el presente trabajo se ha estudiado la hidratación inicial de un cemento portland aditivado con superplastificantes de diferente naturaleza. Dichos aditivos fueron: uno basado en melaminas sintéticas, otro en copolímeros vinílicos y otro en policarboxilatos. La dosificación de los dos primeros se fijó constante en $1 \%$ en peso con relación al cemento, mientras que para el tercero se evaluó, también, la influencia de la dosificación. tomando proporciones desde el 1\% hasta el 0,3\%. Las pastas obtenidas se estudiaron por: calorimetria de conducción, DRX y FTIR. También se determinó la fluidez de la pasta a través del ensayo del "Minislump". Los resultados obtenidos han indicado que: a) Los aditivos superplastificantes utilizados, independientemente de su naturaleza y para el aditivo basado en policarboxilados de su dosificación, retardan la hidratación de los silicatos (especialmente de la fase alita). b) La formación de etringita se ve afectada por la naturaleza del superplastificante. c) Se ha establecido la relación existente entre la dosificación del aditivo basado en policarboxilatos y el tiempo en el que aparece el máximo de la señal de aceleración. Obteniéndose una relación lineal $(y=11,03+$ 16,05x). A partir de estos resultados es posible, en función de la dosificación del aditivo, conocer el tiempo en el que se produce la precipitación masiva de productos, así como los tiempos de fraguado. También se ha comprobado que el calor desprendido como consecuencia de las de las reacciones, asociadas a esta etapa, es independiente de la naturaleza y dosificación del aditivo, poniendo de manifiesto que las reacciones que se producen son las mismas.

\section{SUMMARY}

Early hydration of portland cement with superplasticizer admixtures of different nature has been studied. These admixtures were: one based on melanine synthetic, other based on vinyl copolymer and other based on polyacrylate copolymers. The dosage of the formers were constant $1 \%$ weigth of cement) and for the third, the influence of admixture dosage was also evaluated, giving dosage values among 1-0.3\%. The pastes obtained were studied by conduction calorimetry, XRD and FTIR. Also the apparent fluidity was determined by "Minislump" test. The main results obtained were: a) superplasticizers admixtures used, regardless of their nature and for the polycarboxilate one the dosage, retard the silicate hydration (specially, alite phase). b) The ettringite formation is affected by the nature of the admixture. c)A relationship between the dosage of admixture based on polycarboxilates and the time at the acceleration has been established. A lineal relation $(y=$ $11.03+16.05 x)$ was obtained. From these results is possible to know, in function of dosage admixture, the time when the masive hydration products and the setting times are produced. Also the total heat releases in these reactions is independent of the nature and dosage of admixture, saying that in all cases the reactions are the same. 


\section{INTRODUCCIÓN}

La utilización de superplastificantes en la elaboración de hormigones, ya sean éstos de características especiales o no, es, hoy en día, una práctica habitual. Los superplastificantes se emplean con el objeto de aumentar significativamente la trabajabilidad para una relación a/c dada, producir una reducción considerable de la relación $\mathrm{a} / \mathrm{c}$ para una determinada trabajabilidad, y obtener simultáneamente ambos efectos.

Es un grupo de aditivos relativamente reciente, aunque se conocen formulaciones con capacidad dispersante desde los años 30. Estos aditivos se denominan también como reductores de agua de alta actividad, y son utilizados en mayor proporción que los convencionales plastificantes. Al igual que éstos, los superplastificantes son surfactantes que se caracterizan por poseer actividad superficial debido a que en su composición química incluyen grupos hidrofilicos y grupos hidrofóbicos. Los superplastificantes son polímeros de alto peso molecular solubles en agua. La solubilidad del aditivo está asegurada por la presencia de hidroxilos y grupos sulfonato o carboxílicos unidos a la cadena orgánica, la cual es normalmente aniónica (1).

De modo general, se puede considerar que hay cuatro grandes categorías de superplastificantes que se utilizan en la industria del hormigón. Estos son (2):

- Condensados de melamina-formaldehido sulfonado (SMF). Estos aditivos fucron desarrollados en Alemania en los años 60. El grado de condensación está habitualmente en el rango de 50-60, de ahí que los pesos moleculares sean del orden de 12.000-15.000.

. Condensados de naftalen-formaldehido sulfonados (SNF). Fueron desarrollados en Japón, en 1963. En este aditivo el grado de condensación es de 5-10 y el peso molecular está en el rango de 1.000-2.000.

. Lignosulfonatos modificados. Son matcriales purificados en los que se han eliminado todas las impurezas de carbohidratos. Tienen altos pesos moleculares. Estos lignosulfonatos obtenidos en los procesos de refinamiento se aproximan a los condensados en términos de comportamiento, aunque tienen una mayor tendencia a retener aire.

- Otros polímeros sintéticos, tales como poliésteres, carboxílicos, vinilos, polímeros hidroxilados y dispersiones de copolímeros, bien aislados o en combinación.

Los tres primeros se caracterizan por tener el mismo grupo funcional, el grupo sulfonato. En la Figura 1 se presentan las unidades moleculares de estos aditivos (2,
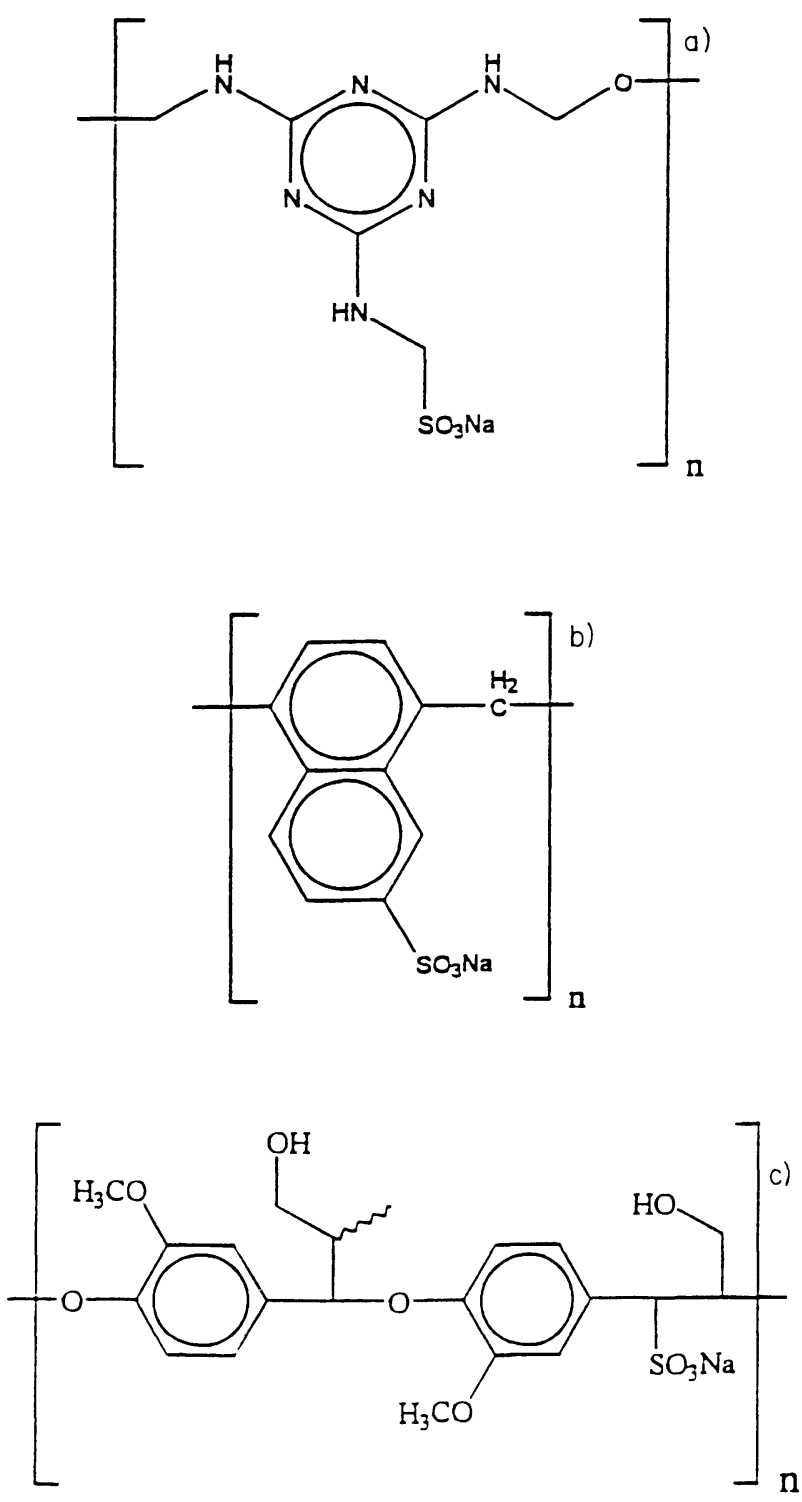

Figura 1.- Unidades moleculares de: a) SMF, b) SNF, c) Lignosulfonatos modificados.

3). Mas recientemente, se han desarrollado, y continúa haciéndose en la actualidad, otros polímeros surfactantes en los que los grupos funcionales responsables de su solubilidad en agua son del tipo carboxílico o hidróxilo. Dentro de éstos destacan los policarboxilatos o poliacrilatos que tienen unas propiedades dispersantes mayores que los polímeros basados en grupos sulfonatos. En la Figura 2a se muestra la unidad molecular genérica de copolímeros de poliacrilato (3). En los últimos años han proliferado formulaciones de copolímeros polifuncionales con gran capacidad dispersantes y fluidificante. Una unidad molecular de este tipo de copolímeros con grupos vinílicos, se muestra en la Figura $2 \mathrm{~b}(3)$.

La acción dispcrsante de los aditivos superplastificante esta producida por la adsorción de las moléculas del aditivo en la superficie de los granos de cemento durante 

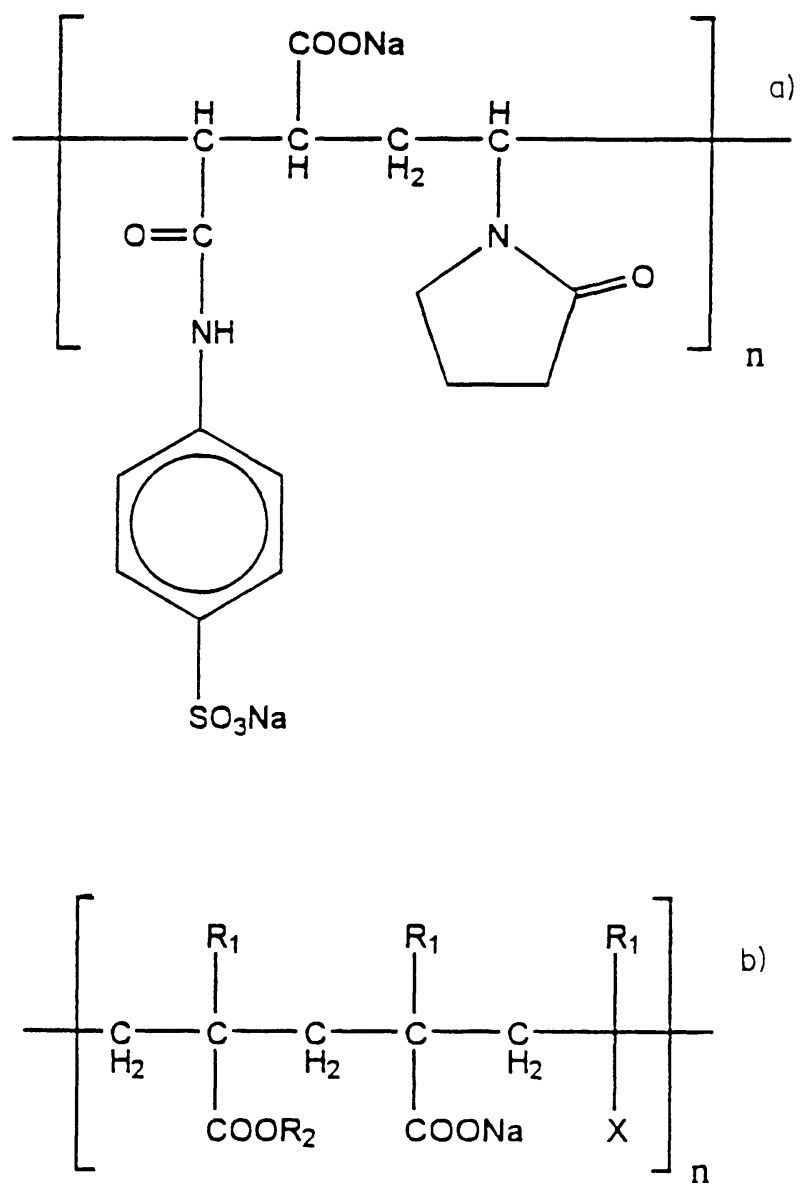

Figura 2.- Unidades moleculares de: a) Copolímero con grupos vinilo, b) Copolímeros de poliacrilato.

las reacciones de hidratación inicial. Sin embargo, no existe un conocimiento suficiente en torno a la naturaleza química de dicha adsorción, a la interacción electrostática y al impedimento estérico que se produce.

Los superplastificantes afectan a la hidratación del cemento porltand, ya que por una parte pueden retrasarla y por otra pueden afectar a la morfología y microestructura de los productos de reacción. De manera general, se asume que la influencia de los superplastificantes en la hidratación del cemento afecta a varios factores (4):

-Las moléculas de superplastificante impiden la difusión del agua y de los iones $\mathrm{Ca}^{2+}$ en la interfase disolucióncemento.
-Los iones $\mathrm{Ca}^{2+}$ forman complejos con las moléculas del superplastificante inhibiendo la nucleación y crecimiento de las especies ricas en $\mathrm{Ca}$.

-La fucrte acción dispersiva de estos aditivos altera la cinética de formación y morfología de los productos de reacción.

Este efecto es variable dependiendo del tipo de aditivo superplastificante. En el presente trabajo se estudia la hidratación inicial de un cemento portland aditivado con superplastificantes de diferente naturaleza.

\section{PARTE EXPERIMENTAL}

Se utilizó un cemento portland tipo CEM I 42,5, cuyo análisis químico se da en la Tabla 1. La caracterización mineralógica realizada sobre este cemento por Difracción de Rayos X (DRX) y Espectroscopía Infrarroja (FTIR) ha mostrado que el cemento contiene alita como fase mayoritaria, junto con belita, $\mathrm{C}_{3} \mathrm{~A}$ y fase ferrito. El sulfato se encontraba en forma de yeso. La composición potencial del clínker, de acuerdo a Bogue (\% en peso), sería: $61,6 \%$ $\mathrm{C}_{3} \mathrm{~S}, 8 \% \mathrm{C}_{2} \mathrm{~S}, 8 \% \mathrm{C}_{3} \mathrm{~A}, 13,7 \% \mathrm{C}_{4} \mathrm{AF}$.

Se emplearon 3 aditivos de distinta naturaleza. Dichos aditivos fueron:

Aditivo A: basado en melaminas sintéticas modificadas Aditivo B: basado en copolímeros vinílicos modificados Aditivo C: basado en policarboxilato modificado

Se prepararon pastas de cemento, con relación $a / c=0,4$, en las que el aditivo se adicionó al agua de amasado. La dosificación de los aditivos fue la siguiente: Aditivo $\mathrm{A}=$ $1 \%$ en peso de cemento, aditivo $\mathrm{B}=1 \%$ en peso de cemento y aditivo $\mathrm{C}=0,3 ; 0,5 ; 0,7$ y $1 \%$ en peso de cemento. También se prepararon pastas exentas de aditivo como muestras de referencia.

Sobre las diferentes pastas se realizó una calorimetría de conducción con el objeto de monitorizar la velocidad de desprendimiento de calor debido a las distintas reacciones de hidratación con el tiempo. Se determinaron los tiempos de fraguado a través del ensayo de la Aguja de Vicat.

TABLA 1

Composición química del cemento, superficie específica y tiempos de fraguado

\begin{tabular}{|c|c|c|c|c|c|c|c|c|c|}
\hline P.C. & R.I. & $\mathrm{SiO}_{2}$ & $\mathrm{Al}_{2} \mathrm{O}_{3}$ & $\mathrm{Fe}_{2} \mathrm{O}_{3}$ & $\mathrm{CaO}$ & $\mathrm{MgO}$ & $\mathrm{SO}_{3}$ & $\mathrm{Na}_{2} \mathrm{O}$ & $\mathrm{K}_{2} \mathrm{O}$ \\
\hline 0.79 & 0.17 & 18.95 & 5.89 & 4.50 & 63.83 & 2.21 & 2.85 & 0.29 & 0.75 \\
\hline \multicolumn{10}{|c|}{ Blaine: $403,7 \mathrm{~m}^{2} / \mathrm{Kg}$} \\
\hline
\end{tabular}

* Pérdida por calcinación 
A las edades de $3 \mathrm{~h}$ y $46 \mathrm{~h}$, las pastas fueron congeladas con acetona y alcohol. Las muestras resultantes fueron analizadas por Difracción de Rayos X (DRX), y Espectroscopía Infrarroja por Transformada de Fourrier (FTIR).

Se determinó la variación en la fluidez de la pasta mediante cl ensayo del "Minislump", a las edades de 5, 15, $30 \mathrm{y}$ 60 minutos. Para este ensayo, las pastas fueron preparadas en amasadora durante 3 minutos, y vertidas sobre un molde troncocónico de $19 \times 38,1$ × 57,2 mm. Después de 10 golpes en la mesa de sacudidas, se midió el diámetro de la pasta en dos direcciones. El valor final corresponde con la media aritmética de dichas mediciones.

\section{RESULTADOS Y DISCUSIÓN}

La hidratación del cemento portland es un proceso exotérmico por lo que la velocidad de desprendimiento de calor es una medida de la velocidad del proceso. Se pueden considerar cinco ctapas diferenciadas en las curvas de velocidad de desprendimiento de calor de un cemento hidratado. La primera etapa corresponde con una señal muy intensa debida a la hidratación inicial en la superficie de las partículas de cemento. La duración de esta etapa es muy corta (alrededor de 20 minutos) y finaliza cuando se inicia la segunda etapa denominada "período de inducción", en la que la velocidad de desprendimiento de calor es muy baja. Este período se alarga uno o dos horas, tiempo durante el cual la pasta es trabajable. Después de este tiempo, la capa superficial de productos que recubre los granos de cemento se rompe y/ o también los cristales de portlandita crecen, lo que provoca que la velocidad de desprendimiento de calor aumente y se produzca el fraguado, apareciendo en las curvas calorimétricas un segundo pico. Esta señal, corresponde con la tercera etapa conocida con el nombre de aceleración. Esta señal se produce alrededor de las 10 horas y se asocia a la precipitación masiva de productos de reacción (basicamente gel CSH y portlandita). Después de esta etapa la velocidad de las reacciones disminuye, debido a que el proceso pasa a ser gobernado por la difusión, dando lugar a la cuarta etapa denominada de desaceleración. En algunos cementos, se observa un tercer pico en las curvas de velocidad de desprendimiento de calor, entre las 18 y las 30 horas, que se asigna como a la reacción final del $\mathrm{C}_{3} \mathrm{~A}$ con el yeso. La última etapa es la finalización en la que las reacciones transcurren ya a muy baja velocidad (5).

En la Figura 3 se muestran las curvas de velocidad de desprendimiento de calor de las distintas pastas estudiadas. En la Tabla 2 se presentan de manera resumida los datos calorimétricos asociados principalmente a la etapa de accleración. Analizando estos resultados se observa que la curva calorimétrica de la pasta que contiene el
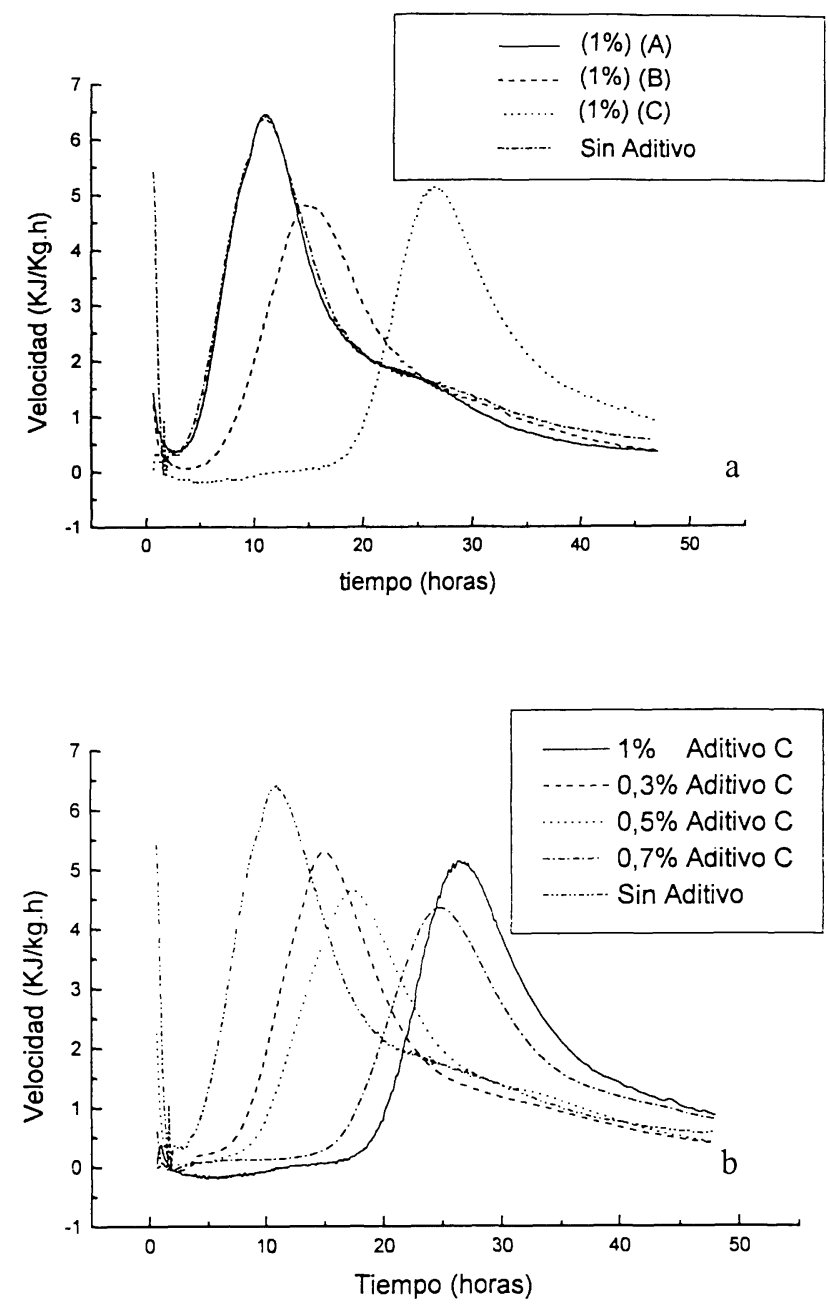

Figura 3.- Curvas calorimétricas de las diferentes pastas.

aditivo A (basado en melaminas sintéticas) es prácticamente idéntica a la de la pasta exenta de aditivos. Por el contrario con el aditivo $\mathrm{B}$ (copolímeros vinílicos) y muy especialmente con el aditivo $\mathrm{C}$ (policarboxilatos) se produce un notable retraso en las reacciones de hidratación, que se manifiestan en las curvas de velocidad de desprendimiento de calor por un alargamiento del período de inducción y una disminución en la velocidad de desprendimiento de calor debida a la señal de aceleración. Ese alargamiento puede llegar a ser hasta de $18 \mathrm{~h}$ frente a las 2-3 h en la pasta no aditivada; la disminución en la velocidad de la señal de aceleración pucde llegar a ser hasta del 30\%. Sin embargo, esa disminución no comporta menor calor desprendido, ya que la integración de dicha señal de aceleración supone en todos los casos un calor desprendido del orden de $200 \mathrm{~kJ} / \mathrm{kg}$.

La influencia de la dosificación de aditivo $\mathrm{C}$ (basado en policarboxilatos) sobre la velocidad de las reacciones se presenta en la Figura 3b. De dicha figura y de los datos calorimétricos de la Tabla 2 se deduce que al incrementarse la dosificación del aditivo se retrasan las reacciones de hidratación. En la Figura 4 se representa la relación 
TABLA 2

Datos calorimétricos

\begin{tabular}{|c|c|c|c|c|c|c|c|}
\cline { 2 - 7 } \multicolumn{1}{c|}{} & $\begin{array}{c}\text { SIN } \\
\text { ADITIVO }\end{array}$ & $\begin{array}{c}\mathbf{A} \\
\mathbf{1 \%}\end{array}$ & $\begin{array}{c}\mathbf{B} \\
\mathbf{1 \%}\end{array}$ & $\begin{array}{c}\mathbf{C} \\
\mathbf{1 \%}\end{array}$ & $\begin{array}{c}\mathbf{C} \\
\mathbf{0 , 7} \%\end{array}$ & $\begin{array}{c}\mathbf{C} \\
\mathbf{0 , 5}\end{array}$ & $\begin{array}{c}\mathbf{C} \\
\mathbf{0 , 3}\end{array}$ \\
\hline $\begin{array}{c}\text { CALOR TOTAL } \\
\text { (Kj/h) }\end{array}$ & 345 & 322 & 272 & 251 & 243 & 262 & 269 \\
\hline $\begin{array}{c}\text { VELOCIDAD } \\
\text { SENAL } \\
\text { ACELERACIÓN } \\
\text { (Kj/Kg.h) }\end{array}$ & 6.36 & 6.40 & 4.82 & 5.10 & 4.35 & 4.64 & 5.28 \\
\hline $\begin{array}{c}\text { TIEMPO } \\
\text { SEÑAL } \\
\text { ACELERACIÓN } \\
\text { (h) }\end{array}$ & 11.17 & 11.01 & 15.02 & 26.40 & 24.8 & 17.4 & 15.0 \\
\hline $\begin{array}{c}\text { CALOR ASOCIADO } \\
\text { SEÑAL } \\
\text { ACELERACIÓN } \\
\text { (Kj/h) }\end{array}$ & 220 & 218 & 204 & 202 & 177 & 180 & 177 \\
\hline $\begin{array}{c}\text { TIEMPO INICIO } \\
\text { ACELERACIÓN } \\
\text { (h) }\end{array}$ & 2.86 & 2.57 & 3.88 & 16.80 & 15.3 & 7.8 & 5.9 \\
\hline $\begin{array}{c}\text { TIEMPO FINAL } \\
\text { ACELERACIÓN } \\
\text { (h) }\end{array}$ & 20.36 & 19.60 & 25.84 & 37.40 & 33.5 & 25.9 & 23.4 \\
\hline
\end{tabular}

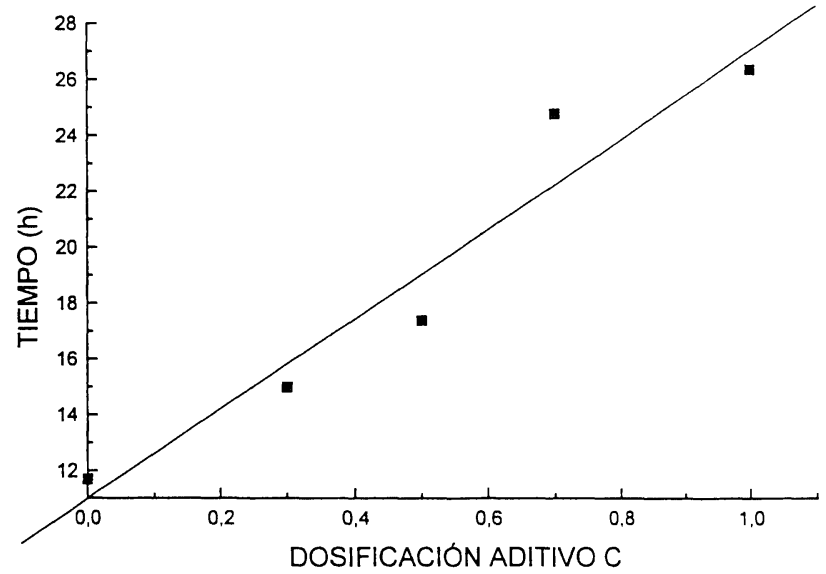

Figura 4.- Influencia de la dosificación de aditivo $\mathrm{C}$ sobre el tiempo del máximo de la señal de aceleración en las curvas calorimétricas.

existente entre la dosificación del aditivo C y el tiempo en el que aparece el máximo de la señal de aceleración. Como puede observarse, la relación lineal [1] es muy buena, con un $\mathrm{R}^{2}=0,9657$, permitiendo predecir, en función de la dosificación del aditivo, cl tiempo al cual la precipitación masiva de productos se va a producir, así como los tiempos de fraguado.

$$
y=11,03+16,05 x
$$

Nuevamente se comprueba, por la integración de la señal calorimétrica de aceleración, que el calor desprendido como consecuencia de las reacciones, asociadas a esta etapa, son independientes de la dosificación del aditivo, iguales en todas las pastas. Es decir, las reacciones que se producen son las mismas.

El retraso en las reacciones de hidratación del cemento por efecto de los aditivos superplastificantes se plasma también en los tiempos de fraguado de las pastas, como puede ser observado en la Tabla 3. No sólo se retrasa el tiempo de inicio de fraguado sino que también se alarga el final del fraguado, esto es más marcado con el aditivo $\mathrm{C}$ en una dosificación del 1\% que en los otros dos restantes aditivos. La variación en la dosificación del aditivo $\mathrm{C}$ también se manifiesta en alteraciones en los tiempos de fraguado.

A partir de las distintas curvas calorimétricas obtenidas, se seleccionaron dos tiempos de reacción para la caracterización mineralógica de las pastas: $3,5 \mathrm{~h}$, en el inicio de la señal de aceleración y 46h, tiempo al cual las reacciones de hidratación que explican la señal de aceleración ya se han completado. Dicha caracterización se realizó por DRX y FTIR.

En la Figura 5 se muestran los difractogramas de las distintas pastas a las dos edades de estudio. Analizando 
TABLA 3

Tiempos de fraguado

\begin{tabular}{|c|c|c|c|c|c|c|c|}
\hline $\begin{array}{c}\text { TIEMPOS } \\
\text { DE } \\
\text { FRAGUADO }\end{array}$ & $\begin{array}{c}\text { SIN } \\
\text { ADITIVO }\end{array}$ & $\mathbf{1 \% A}$ & $\mathbf{1 \% B}$ & $\mathbf{1 \% C}$ & $\mathbf{0 , 7 \%} \mathrm{C}$ & $\mathbf{0 , 5 \% C}$ & $\mathbf{0 , 3 \% C}$ \\
\hline INICIO & $3 \mathrm{~h} 18 \mathrm{~min}$ & $4 \mathrm{~h} 5 \mathrm{~min}$ & $6 \mathrm{~h} 45 \mathrm{~min}$ & $11 \mathrm{~h} 18 \mathrm{~min}$ & $9 \mathrm{~h} 9 \mathrm{~min}$ & $7 \mathrm{~h} 16 \mathrm{~min}$ & $5 \mathrm{~h} 27 \mathrm{~min}$ \\
FINAL & $4 \mathrm{~h} 58 \mathrm{~min}$ & $6 \mathrm{~h} 15 \mathrm{~min}$ & $8 \mathrm{~h} 45 \mathrm{~min}$ & $18 \mathrm{~h} 38 \mathrm{~min}$ & $18 \mathrm{~h} 9 \mathrm{~min}$ & $1 \mathrm{lh} 56 \mathrm{~min}$ & $9 \mathrm{~h} 57 \mathrm{~min}$ \\
\hline
\end{tabular}
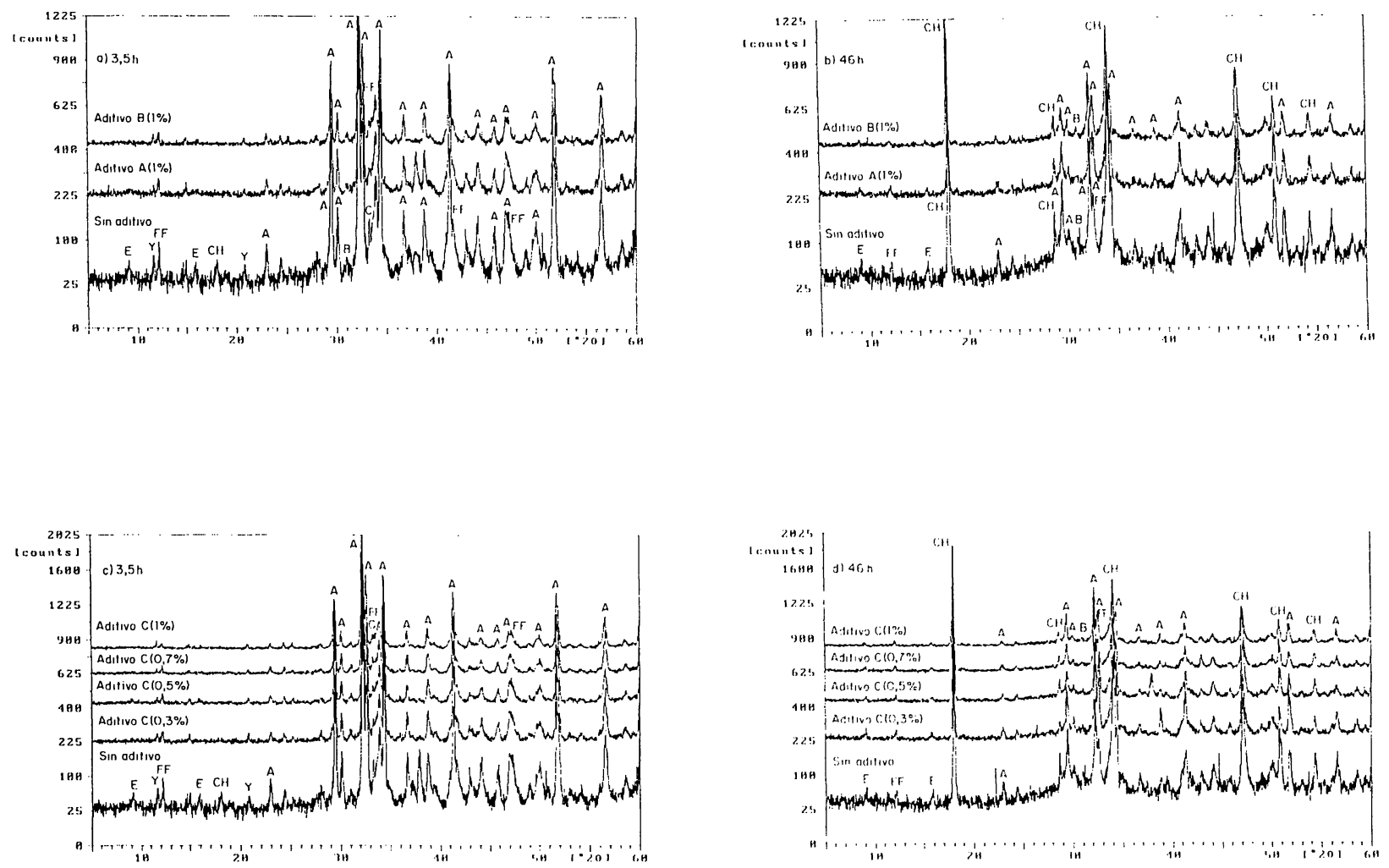

Figura 5.- Resultados de DRX.

estos difractogramas y comparando el de la pasta no aditivada con aquéllas que contienen los aditivos, a las 3,5 horas de reacción, se deduce que:

-La pasta que contiene el aditivo A presenta en su composición etringita como único producto cristalino de reacción, no llegándose a detectar la presencia de $\mathrm{Ca}(\mathrm{OH})_{2} \mathrm{O}$ portlandita.

-La pasta que contiene el aditivo B no presenta en su composición ni etringita ni portlandita, permaneciendo inalteradas la mayor parte de las fases anhidras (alita, belita, $\mathrm{C}_{3} \mathrm{~A}$ y $\mathrm{C}_{4} \mathrm{AF}$ ).

-Las pastas que contienen el aditivo $\mathrm{C}$ presentan diferente composición dependiendo de su dosificación. Con 0,3 y $0,5 \%$, se observa la presencia de etringita, con $0,7 \%$ se detecta esta fase aunque en muy pequeña proporción y finalmente, con un 1\% la etringita no es observada. En ninguna de las pastas activadas con el aditivo $\mathrm{C}$ se encuentra portlandita.

La no presencia de portlandita en ninguna de las pastas aditivadas a las 3,5 horas de reacción está indicando un retardo en la reacción de hidratación del $\mathrm{C}_{3} \mathrm{Sss}$ (alita). A las 46 horas de reacción se detecta etringita y portlandita en todas las pastas.

La Figura 6 muestra la variación en el $\mathrm{n}^{\circ}$ de cuentas de las líneas de difracción situadas en $2 \theta=18,02^{\circ}$ (identificativa de la portlandita) y en $2 \theta=32,26^{\circ}$ (perteneciente a la fase alita) a las dos edades de estudio. Estas gráficas muestran que la disminución de las cuentas debidas a la alita de 3,5 a $46 \mathrm{~h}$ es, en todas las pastas, muy similar. Unicamente la pasta con el aditivo $\mathrm{C}$ en un porcentaje (ver Fig. 6b) del 1\% en peso presenta a las $3,5 \mathrm{~h}$ mayor cantidad de fase alita. 

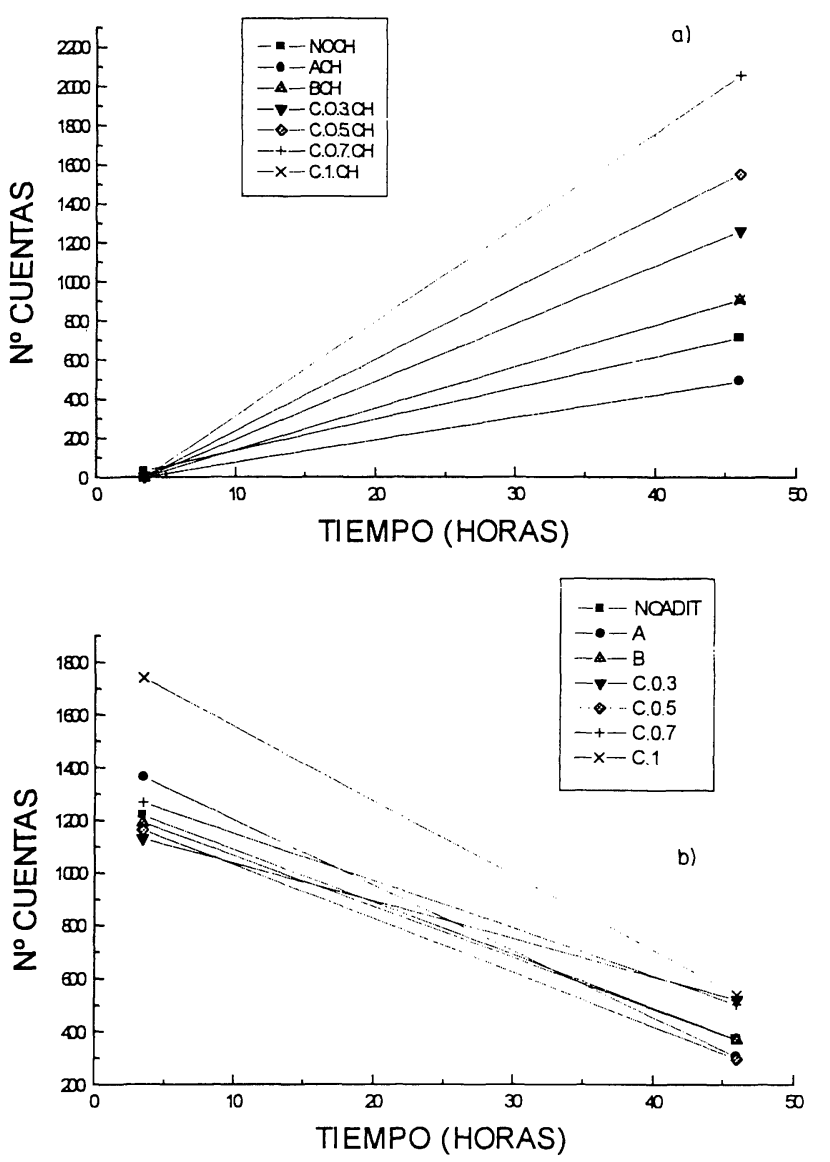

Figura 6.- Evolución con el tiempo del $n^{\circ}$ cuentas de lineas de difracción identificativas: a) $\mathrm{Ca}(\mathrm{OH})_{2}$, b) $\mathrm{C}_{3} \mathrm{Sss}$.

La portlandita (Figura 6a) no se detecta en ninguna de las pastas aditivadas a las $3,5 \mathrm{~h}$ de reacción, sin embargo, sí se observa en cantidades muy variables a las $46 \mathrm{~h}$. En general, las pastas que contienen cl aditivo $C$ presentan a esta edad de estudio contenidos más elevados de portlandita que las restantes pastas.

Los espectros de FTIR de las pastas se presentan en la Figura 7. El análisis de estos espectros confirma los resultados ya indicados de DRX. En los espectros de las pastas aditivadas, a las 3,5 h de hidratación, la banda $v_{3}(\mathrm{Si}-\mathrm{O})$ del $\mathrm{C}_{3}$ Sss (alita) situada hacia $925 \mathrm{~cm}^{-1}$, no se desplaza hacia frecuencias mayores, lo que indicaría una polimerización de los tetraedros de silicato para formar gel $\mathrm{CSH}$, ni se observa la presencia de la banda a $3.640 \mathrm{~cm}^{-1}$ indicativa de la vibración de tensión de los grupos $\mathrm{OH}$ del $\mathrm{Ca}(\mathrm{OH})_{2}$ o portlandita. Sin embargo, en la pasta no aditivada dicho desplazamiento se produce ligeramente, observándose la banda identificativa de la portlandita. Estos resultados confirman el retraso en la hidratación de los silicatos ya indicada en las pastas que contienen aditivo. También se identifica en la pasta sin aditivar la presencia de etringita por la absorción situada entre $1.100-1.150 \mathrm{~cm}^{-1}$ de grupos $\mathrm{SO}_{4}$, y la variación de la absorción hacia $840-850 \mathrm{~cm}^{-1}$ asignable a vibraciones Al-O.

A las 46 horas, se observa en todos los espectros el desplazamiento de la banda $v_{3}(\mathrm{Si}-\mathrm{O})$ hacia frecuencias mayores $\left(976-980 \mathrm{~cm}^{-1}\right)$, indicando la formación de gel CSH. Los aluminatos anhidros en los cementos con aditivo han reaccionado a esta edad. Sin embargo hay una absorción de mayor intensidad hacia $720-840 \mathrm{~cm}^{-1} \mathrm{en} \mathrm{la}$ muestra sin aditivo que indica una mayor formación de ctringita en esta muestra que en las pastas que contienen aditivo. A través de esta técnica no se han observado diferencias significativas en los espectros IR de las pastas que contienen dosificaciones variables del aditivo $\mathrm{C}$.

Los resultados presentados en este trabajo han demostrado que todos los aditivos superplastificantes utilizados, independientemente de su naturaleza y para el aditivo $\mathrm{C} \mathrm{de}$ su dosificación, retardan la hidratación de los silicatos (especialmente de la fase alita). Ello es debido a la adsorción del polímero en los granos de cemento formando una especie de "picl" alrededor de dichos granos impidiendo el acceso de las moléculas de agua a las partículas de cemento, y también por la formación de complejos entre los iones $\mathrm{Ca}^{2+}$ formados en la hidratación inicial y los aniones de los polímeros, que afectan a la nucleación y precipitación de $\mathrm{Ca}(\mathrm{OH})_{2}(4,6)$.

Únicamente con el aditivo A, basado en melaminas, y a dosificaciones $<0,7 \%$ del aditivo $\mathrm{C}$ se detectó, a las 3,5 h de reacción, la fase etringita. El efecto de los superplastificantes en la formación y estabilización de la etringita es un aspecto altamente discutido, ya que los resultados obtenidos por diferentes autores son muy diversos. Ramachandra (7), estudiando mezclas de $\mathrm{C}_{3} \mathrm{~A}$ yeso con aditivos SMF y SNF comprobó que la velocidad de formación de la etringita se aceleraba en presencia de ambos superplastificantes, sin embargo, la conversión de etringita a monosulfoaluminato se retrasaba o inhibía. Sin embargo, otros autores (8) apuntan resultados distintos ya que obtienen retrasos en la formación de etringita. Previo a la formación de gel CSH como consecuencia de la hidratación de las fases silicato, se produce la formación y precipitación de los cristales de etringita en forma de agujas prismáticas. Algunos autores (4) indican la posibilidad de adsorción de la parte aniónica del superplastificante sobre la superficic de las partículas de etringita, inhibiendo su precipitación e impidiendo cl acceso de agua a las partículas de $\mathrm{C}_{3} \mathrm{~A}$. Otros autores apuntan a que esa adsorción se produce sobre un precursor de la etringita (9). En el presente trabajo se ha comprobado, que en igualdad de condiciones, la formación de etringita varia dependiendo del tipo de superplastificante. Ese hidrato se forma cuando el aditivo es del tipo SMF, aunque en menor cantidad que en la pasta exenta de aditivo, y no se forma con el aditivo basado en copolímeros vinílicos, ni a altas concentraciones del aditivo basado en 

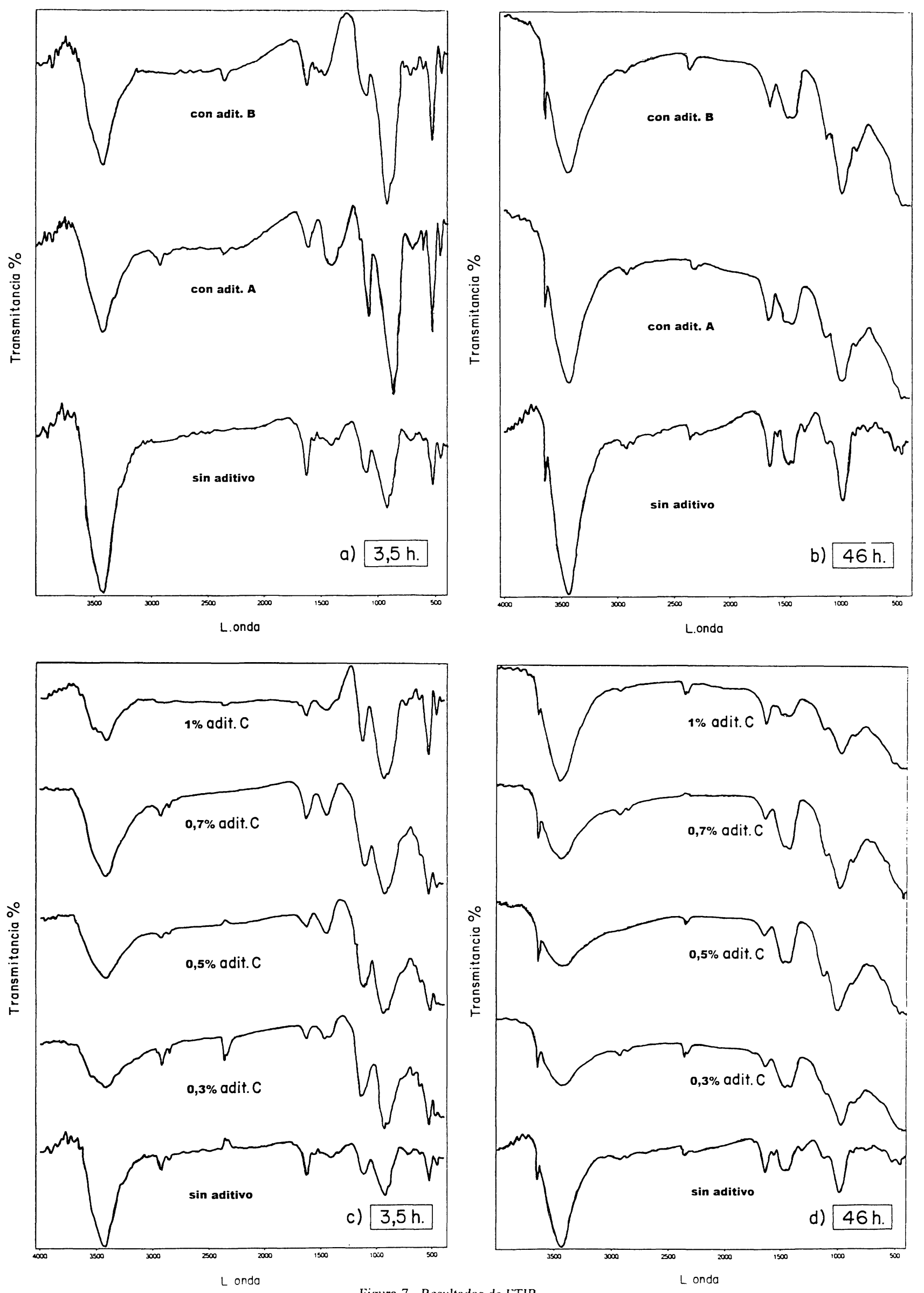

Figura 7.- Resultados de FTIR. 


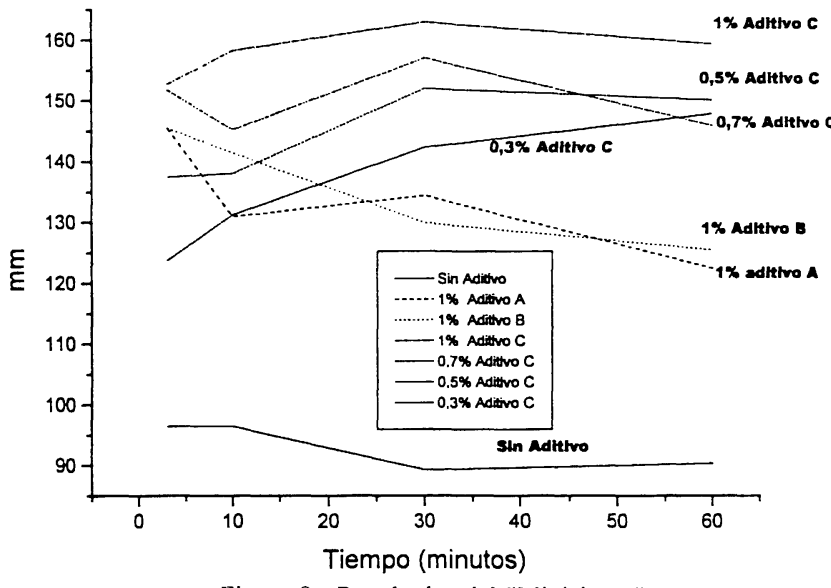

Figura 8.- Resultados del "Minislump".

carboxilatos. La diferente naturaleza del aditivo influye sobre la adsorción y por tanto sobre la formación de ctringita, explicando en parte la controversia existente. Más investigaciones son requeridas.

Finalmente, se estudió la variación de la fluidez de las pastas a través del ensayo del "Minislump". En la Figura 8 se presentan los resultados obtenidos. El aditivo $\mathrm{C}$ es $\mathrm{cl}$ que confiere a la pasta la mayor fluidez. Con los aditivos A y $\mathrm{B}$ el incremento de la fluidez con respecto a la pasta sin aditivo es del orden de un $30 \%$, mientras con el aditivo $\mathrm{C}$ ese incremento se sitúa en torno al 50-60\%. No sólo con el aditivo $\mathrm{C}$ se obtienen mayores dispersiones sino quc, además, se mantienen durante más tiempo.

\section{CONCLUSIONES}

Las principales conclusiones de este estudio son:
-Los aditivos superplastificantes utilizados, independientemente de su naturaleza y para el aditivo basado en policarboxilados de su dosificación, retardan la hidratación de los silicatos (especialmente de la fase alita).

-La formación de etringita se ve afectada por la naturaleza del superplastificante. Ese hidrato se forma a edades tempranas cuando el aditivo es del tipo SMF, en menor cantidad que en la pasta exenta de aditivo, y no se forma con el aditivo basado en copolímeros vinílicos, ni a altas concentraciones del aditivo basado en carboxilatos. La diferente naturaleza del aditivo influye sobre la adsorción del mismo en las fases del cemento, afectando a la formación de etringita.

-Se ha establecido la relación existente entre la dosificación del aditivo basado en policarboxilatos y el tiempo en el que aparece el máximo de la señal de acelcración. Obtenićndose una relación lineal $(\mathrm{y}=11,03$ $+16,05 x)$. A partir de estos resultados es posible, en función de la dosificación del aditivo, conocer el tiempo al cual se produce la precipitación masiva de productos, así como los tiempos de fraguado. También se ha comprobado que el calor desprendido como consecuencia de las reacciones, asociadas a esta etapa, es independiente de la naturaleza y dosificación del aditivo, indicando que las reacciones que se producen son las mismas.

\section{AGRADECIMIENTOS}

Los autores quieren agradecer a la CICYT por la financiación del proyecto MAT 98-0792, sin la cual no habría sido posible la realización de este trabajo. Los autores también agradecen a J. Morales, A. Gil y J. L.García por su colaboración en la ejecución de los distintos ensayos.

\section{BIBLIOGRAFÍA}

(1) J. Roncero, R. Gettu. "Aditivos superfluidificantes para hormigones de altas prestaciones". IV Simposio ANFAH. Especialidades Químicas para la Construcción.(1998)

(2) R.M. Edmeades, P.C. Hewlett. "Cement Admixtures". LEA'S Chemistry of Cement and Concrete. V Edition. Ed. P.C. Hewlett (Arnold), p. 589(1998)

(3) V.S. Ramachandran, V.M: Malhotra, C. Jolicoeur, N. Spiratos. "Superplasticizers: Properties and applications in concrete". CANMET Ed.pp. 45-48 (1998)

(4) M.Y.A. Mollah, W.J. Adams, R. Schennach, D.L. Cocke. "A review of cement-superplasticizer interactions and their models". Avd. Cem. Res., 12, nº 4, pp. 153-161 (2000)

(5) A.M. Neville. "Properties of Concrete". Fourth Edition (Longman), p. 12 (1995)

(6) N.B. Singh, R.Sarvahi, N.P. Singh. "Effect of superplasticizers on the hydration of cement”. Cem. Concr. Res. Vol 22, pp. 725-735 (1992)

(7) V.S. Ramachandra. "Adsorption and hydration behaviour of tricalcium aluminate-water and tricalcium aluminate-gypsum-water systems in the presence of superplasticizers". J. Am. Concr. Inst. 80, pp. 235-241 (1983)

(8) F. Massazza, U. Costa. "Effect of superplasticizers on the C 3 A hydration". 7th Int. Congr. Chem. Cem, Vol 5, pp. 529-534 (1980)

(9) V.T. Yilmaz, F.P. Glasser. "Influence of sulphonated melanine formaldehyde superplasticizers on cement hydration and microstructure". Adv. Cem. Res. Vol 2, No7, pp. 111-119(1989) 